\title{
Coping with cross-reactive carbohydrate determinants in allergy diagnosis
}

FriedRICH ALtMANN

Department of Chemistry, University of Natural Resources and Life Sciences, Vienna, Austria

Key words cross-reactive carbohydrate determinants CCD - glycoproteins - core- $a-$

1,3-fucose a-1,3-galactose alpha-gal

\section{Submitted}

June 26, 2015

\section{Accepted}

August 26, 2015

\section{Abstract}

A relevant proportion of allergy diagnosis is accomplished by in vitro determination of specific immunglobulin E (sIgE) to extracts from suspected allergens. Such extracts inevitably contain glycoproteins, which may react with patients' IgE. In the case of plant and insect allergens, the relevant epitope structure is an $\alpha-1,3$-fucose on the Asn-linked sugar residue of so-called $\mathrm{N}$-glycans. Due to their wide distribution, $\mathrm{N}$-glycans carrying this epitope are known as "cross-reactive carbohydrate determinant(s)" (CCD[s]). About 15 years of awareness allow the conclusion that anti-CCD IgE does not cause noticeable clinical symptoms. In consequence, diagnostic results arising from CCD reactivity must be rated as false positives. With up to $30 \%$ of CCD reactive patients, this can be regarded as a serious problem.

Another cross-reactive carbohydrate determinant became notorious as a potential cause of anaphylactic reactions to a recombinant glycoprotein drug carrying $\alpha-1,3$-galactose. This galactose-containing determinant (GalCD, galactose containing crossreactive carbohydrate determinant) was supposed as a trigger for delayed allergic reactions to red meat

\section{Introduction}

The obvious purpose of any diagnostic measure is to obtain an accurate impression of the patient's condition. In case of allergy diagnosis this entails the identification of the true culprit allergen while avoiding to indict harmless allergens. Among the various "mimickers of allergy" [1], protein-linked carbohydrates are a prominent and well defined cause of false-positive reactions. The following chapters will deal with plant/insect fucose-containing CCDs, how they became accepted as being clinically irrelevant, and how CCD-based false-positive in several cases. Thus, $\alpha$-1,3-galactose may have clinical relevance in certain cases - possibly as a result of tick bites. Often, however, GalCDs probably cause false-positive results with milk and meat extracts. No clear evidence for the role of other non-human carbohydrate structures such as $\mathrm{N}$-glycolylneuraminic acid as CCD has been presented so far.

Remedies for sIgE based in vitro diagnosis come in the form of non-glycosylated recombinant allergen components or of specific CCD inhibitors. The high potential of recombinant allergens is optimally realized in the context of component resolved diagnosis using allergen arrays with more than 100 components, whereas CCD inhibitors increase the specificity of conventional extract-based diagnosis. Reagents for the detection and inhibition of CCDs from plants and insects have been developed, whereas tools for GalCDs of milk and meat lag behind.

Cite this as Altmann F. Coping with cross-reactive carbohydrate determinants in allergy diagnosis. Allergo J Int 2016;25:98-105

DOI: $10.1007 / \mathrm{s} 40629-016-0112-6$

results can be avoided. Finally, the possibly more serious role of $\alpha-1,3$-galactose containing GalCDs will be discussed.

\section{History and structures of plant/insect CCDs}

More than 30 years ago, Aalberse and co-workers incubated patients' sera with an unusual array of allergens and supposed allergens (e.g., potato and buckwheat). They observed an almost ubiquitous crossreactivity of some sera [2]. More precisely, these sera reacted with extracts from pollens, vegetable foods, and - noteworthy - also hymenoptera venoms. The 
reactivity of the allergen extracts could be greatly reduced by periodate treatment, which destroys (mainly) terminal sugars in complex carbohydrates. While not absolutely specific, periodate sensitivity is a good indication for the carbohydrate nature of an epitope.

Meanwhile, several reports on cross-reactive IgG antibodies appeared. The most relevant was on an antiserum against horseradish peroxidase [3]. Astonishingly, this serum stained the neuronal chord in Drosophila embryos. The authors defined the epitope in horseradish as an Asn-linked oligosaccharide (N-glycan) with xylose and a fucose linked $\alpha-1,3$ to the innermost GlcNAc residue (now called MMXF) (Fig. 1). Both elements are foreign to mammals, which explains how this N-glycan can be an epitope. A similar structure had been described earlier for pineapple bromelain [4], which is among the few plant glycoproteins available in decent purity and quantity.

The elucidation of the N-glycans of honeybee venom phospholipase revealed core $\alpha-1,3$-fucose as the structural basis for the cross-reactivity between insect and plant allergens [5]. A parallel study exploited the comparable sensitivity of the fucose linkage to acid degradation to substantiate the role of this fucose residue. A quarter of 122 insect venom positive patients reacted with glycopeptides isolated from protease-degraded bromelain as shown by the ability of these glycopeptides to inhibit the binding of IgE to honeybee venom phospholipase [6]. This ability was largely abolished when the glycopeptides were treated with mild acid just to that point when the fucose residues were essentially removed. Apparently, a highly cross-reactive "allergenic" structure was detected. In the following years, a number of trials to proof its biological significance were undertaken.

\begin{tabular}{|c|c|}
\hline \multicolumn{2}{|c|}{ Abbreviations } \\
\hline BAT & Basophil activation test \\
\hline CCD & $\begin{array}{l}\text { Cross-reactive carbohydrate } \\
\text { determinant }\end{array}$ \\
\hline Fuc (F) & Fucose \\
\hline Gal (G) & Galactose \\
\hline GalCD & $\begin{array}{l}\text { Galactose containing cross-reactive } \\
\text { carbohydrate determinant }\end{array}$ \\
\hline GlcNAc (Gn) & $\mathrm{N}$-acetylglucosamine \\
\hline Man (M) & Mannose \\
\hline Neu5Gc (Ng) & N-glycolylneuraminic acid \\
\hline NRL & Natural rubber latex \\
\hline slgE & Specific immunglobulin $\mathrm{E}$ \\
\hline Xyl (X) & Xylose \\
\hline
\end{tabular}

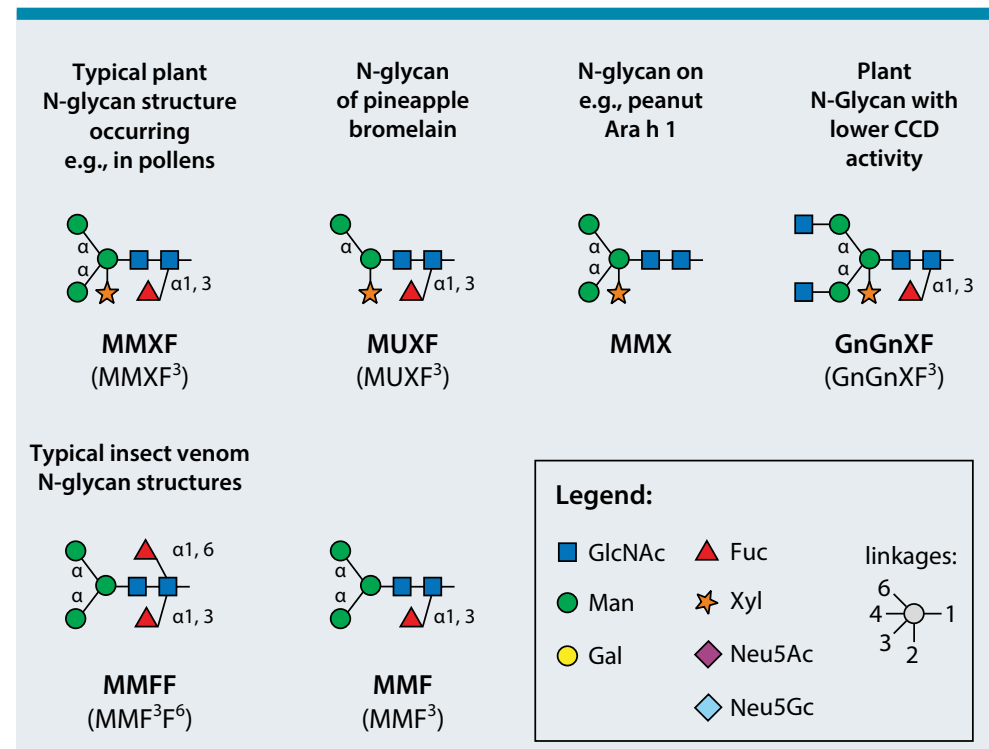

Selection of many possible structures with alpha-galactose
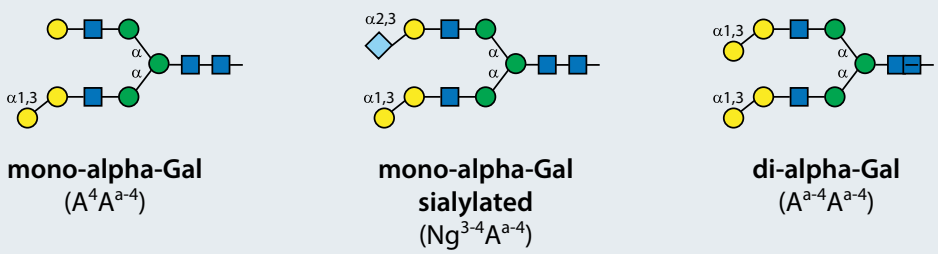

Fig. 1: Selection of relevant cross-reactive carbohydrate determinants. The glycans are composed of galactose (Gal, A), mannose (Man, M), N-acetylglucosamine (GlcNAc, Gn), fucose, (Fuc, F), xylose (Xyl, X), and/or N-glycolylneuraminic acid (Neu5Gc, Ng). Linkages are to be understood as $\beta$, except where indicated as $\alpha$. The abbreviations of structures are according to the proglycan system. Briefly, all terminal sugars are listed starting with the one in the upper left corner and then moving counter-clockwise. In some cases, the linkage of the terminal sugar is unambiguous (e.g., with Man, Xyl, GlcNAc residues), in others the options are specified by superscripts. Further information can be found at www.proglycan.com.

\section{High specificity and affinity of anti-CCD antibodies}

Chemical deglycosylation is a destructive process of limited specificity. Thus, modifications other than the one intended may occur. More evidence for the role of individual sugar moieties could be provided by generating the structure rather than destroying it. This was achieved using recombinant, pure xylosyl- and $\alpha$-1,3-fucosyltransferase and $\mathrm{N}$-glycan acceptors from mammals, which are certainly free of the sugar residues under investigation. Soluble forms of these two glycosyltransferases were expressed in Pichia pastoris and used to modify human transferrin. Before, transferrin was trimmed with sialidase and galactosidase to get the so-called GnGn glycan structure [7]. Thus, glyco-variants of transferrin were generated specifically containing a 
structural feature that was certainly not present on the native transferrin.

These neo-glycoproteins were then used to detect CCD-specific IgG and IgE in patients' sera. Among the patients with multiple grass sensitization, $24 \%$ were positive for $\alpha$-1,3-fucosylated transferrin with or without xylose $\left(\mathrm{MMF}^{3}\right.$ and $\mathrm{MMXF}^{3}$ glycan moiety), whereas none of these sera clearly reacted with the xylosylated transferrin $[7,8]$. Remarkably, rabbit IgG can contain an antibody fraction specific for xylosylated only (MMX) glycans [7, 9].

As a final characterization of human IgE against CCDs, affinity-purified anti-CCD $\operatorname{IgE}$ and anti-CCD IgG were analyzed for their binding affinity to $\mathrm{MMF}^{3}$ and $\mathrm{MMXF}^{3}$ glycans [8]. In great contrast to other carbohydrate proteins such as lectins with their notoriously low binding affinities, the antiCCD antibodies exhibited very high affinity. In the case of IgE, the dissociation constants were comparable to protein allergens. In the case of $\operatorname{IgG}$, the affinity was clearly higher [8].

\section{Biological significance of plant/insect CCDs}

The process leading to the clinical symptoms of allergy can be re-enacted by test monitoring the degranulation of basophilic granulocytes either by measuring released histamine [10], interleukin 4 [11], or vesicle specific protein markers [12]. Such histamine release tests were performed with tomato $\beta$-fructofuranosidase or albumin coupled CCDglycopeptides $[10,11]$ and pointed at biological activity very similar to that of undoubted allergens such as Bet $\mathrm{v} 1$. The obvious conclusion was that core $\alpha$-1,3-fucosylated $\mathrm{N}$-glycans are relevant allergenic epitopes causing clinical symptoms.

Even earlier, van der Veen and co-workers performed histamine release with peanut extracts and sera from peanut allergic patients [13]. With sera that were peanut reactive solely on the basis of CCDs, degranulation only occurred at concentrations much higher than needed for protein based reaction with peanut extract. Due to this result, they concluded that CCDs lack biological significance [13].

However, one may challenge this test system for two reasons: First, the major peanut allergen Ara h 1 is mono-glycosylated and thus cannot perform the cross-linking of Fce receptors required for triggering degranulation. Second, peanut glycoproteins contain almost no fucose [14]. Anti-Xyl IgE never found above baseline - even in peanut allergic patients [Eiwegger T, Altmann F, Vienna, Austria; unpublished results]. Though the science of this work was built on rather uncertain grounds, the conclusion has prevailed. No clear evidence for CCDs provoking allergic reactions has ever been brought forward. As a possible exception the somewhat special case of a tomato allergic patient could be mentioned for whom a xylosylated $\mathrm{N}$-glycan appeared to be part of a peptide epitope [15]. A large study by Adriano Mari, who tested 1,831 subjects of which $23 \%$ reacted towards plant protein $\mathrm{N}$-glycans, did not provide evidence for relevant CCD-based reactions [16]. Of the CCD-positive individuals, $21 \%$ showed but very weak skin reactions when tested with horseradish peroxidase, but not with phospholipase $\mathrm{A}^{2}$ or ascorbate oxidase [16]. Given the up to eight $\mathrm{N}$-glycosylation sites on peroxidase as opposed to one and two on PLA and ascorbate oxidase, this result can be taken as a corroboration of the concept that it requires multivalent binding of an allergen to trigger mast cell degranulation. Moreover, these results confirm the assumption that CCDs do not cause notable clinical symptoms.

Ebo and co-workers came with a different approach to the same conclusion [1]. Much later, the biological significance of anti-CCD IgE was examined by a commercial basophil activation test (BAT), in which CCDs could in fact lead to basophil activation as indicative of biological efficacy [12]. As the patients had been carefully diagnosed, this positive diagnosis against natural rubber latex (NRL) and apple could clearly be rated as falsepositives.

Still unclear is the reason for this essentially complete inability of eliciting manifest allergic symptoms. Jin and co-workers speculated on an inhibitory role of IgG, as the anti-CCD IgG exhibited a higher affinity than IgG against well-known protein allergens [8]. It may well be envisaged that the inevitably frequent uptake of vegetable food (including cereals!) could desensitize against plant $\mathrm{N}$-glycans by a mechanism akin sublingual immune therapy.

It is unknown why certain people develop anti-CCD IgE. A link to hymenoptera stings is often discussed [17], whereas the increased risk for antiCCD IgE development among heavy drinkers supposedly does not explain the whole phenomenon [18]. An interesting link to the physiological function of IgE may be posed by a study, which found increased anti-CCD IgE as a result of parasite infection [19].

\section{False positive diagnosis due to CCDs and possible solutions}

The percentages of allergic individuals sensitized to plant/insect CCDs may be estimated from two large studies, where sera were collected rather unbiased and in which anti-CCD IgE was found in $23 \%$ [16] and $22 \%$ [20] of patient cohorts numbering well over 1,000 individuals. In the young adult cohort, the prevalence reached $30 \%$ [20]. Based on an admittedly only semi-quantitative strip test, most of the reactions were RAST class 2 or higher [20]. For- 
tunately for the patients, their anti-CCD IgE does not bring them any inconvenience.

Already in 1998, Aalberse stated "These results support the concept that the accuracy of serological allergy tests will improve if CCD-related reactivity can be avoided" [21]. However it required the fundamental study of Mari [16] to anchor the clinical insignificance of CCDs in the consciousness of the experts. Mari's conclusions were supported by a study on "mimickers of allergy", which concluded "Sensitization to profilin and/or bromelain-type CCD, caused by pollen (timothy grass, mugwort) or hymenoptera venom allergens, can elicit false-positive IgE antibodies against natural rubber latex and apple“ [1].

A particular problem is the assignment of the culprit insect in the case of apparent double sensitization against bee and wasp venom, which often is solely based on CCD cross-reactivity and only rarely a true double sensitization [22]. Nine years later, the idea of the innocence of CCDs consolidated further and Hemmer wrote "For the time being, we appreciate judging these antibodies as clinically insignificant as a useful hypothesis, ..." [23]. A consistent diagnosis of vespid venom allergy should eliminate the CCD problem, e.g., "by CCD-blocking" [24].

Taken together, CCDs found in virtually all plant allergen extracts and in all hymenoptera venoms are a frequent cause of false-positive diagnostic results in extract-based sIgE determinations.

Four possible remedies for the CCD problem:

1. In vivo testing such as skin-prick test or placebo controlled food challenges.

2. Ex vivo testing such as the basophil activation test, which, however, is not entirely unaffected by CCDs [1].

3. In vitro testing with recombinant allergens. This is increasingly done with the label "component resolved diagnosis" either in single allergen tests or on strips or biochip arrays, the most prominent of which currently is the ImmunoCAP ISAC system (Phadia, Uppsala, Sweden) comprising 112 components from 51 allergen sources. Component resolved allergy diagnosis very often gives a better guidance with regard to the steps to be taken as a consequence of a positive reaction, e.g., because it helps to unveil the primary sensitizing agent $[25,26,27]$. Recombinant allergens are typically produced in Escherichia coli and thus are devoid of any glycosylation. Therefore, there is no CCD problem anymore in theory? However, the ISAC array contains a number of allergen components purified from natural extracts. Indeed, a recent study revealed nPhl p 4, nCyn d 1, nJug r 2, nCry j 1, nCup a 1, nOle e 1 and nPla a 1 (the " $n$ " indicating natural as opposed to recombinant array component) as being recognized by anti-CCD IgE, whereas nGly m 5, nGly m 6, nArt v 1, nAmb a 1, nJug r 1 and nJug r 3 were not [20]. So, even the ImmunoCAP ISAC is prone to deliver false positive results based on CCD-reactive IgE. But for most components, the sentence "... yields the impression of a discrepancy as positive findings in extract-based diagnosis were not verified by the ISAC results." [28] will hold true.

Single allergen ImmunoCAP assays will show the same problem with the above mentioned natural components. Moreover, with CCD-positive sera, ImmunoCAP tends to yield +/- threshold readings for recombinant proteins despite their production in bacteria. The astonishing reason is that the cellulose sponges do not only consist of cellulose but also of some cotton glycoproteins carrying CCD epitopes [29]. The supplier may meanwhile have overcome this drawback.

4. In vitro testing with competitive inhibition with a specific CCD-inhibitor (Fig. 2). Any plant glycoprotein carrying an MMXF or MUXF structure could be used as inhibitor. The glycoprotein should contain several CCD structures to exploit the multivalency effect and it should be very pure to avoid an adverse influence by impurities that may have homologies to the allergens to be investigated. Similarly, the glycoprotein itself must not contain (cross-)reactive protein epitopes. This requirement can be met by proteolytic degradation of the glycoprotein, purification, and finally coupling of the glycopeptide to an immunologically inert carrier.

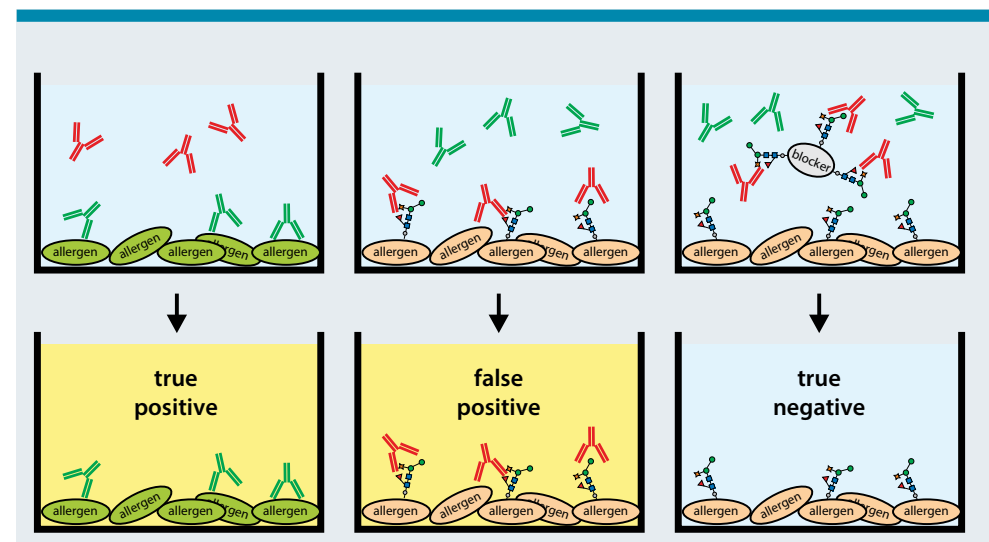

Fig. 2: Principle and effect of competitive inhibition of anti-CCD IgE. The left cup shows a reaction between a protein allergen and its specific lgE. The middle cup was coated with a glycoprotein that cross-reacts with anti-CCD IgE. Both samples give positive results. In the right cup, a polyvalent inhibitor (e.g., the semisynthetic glycoprotein used by Holzweber and coworkers [20]) was added resulting in competitive inhibition with coat allergen for the binding of CCD-specific IgE and thus in a - justifiably - negative result. 
An example for the use of natural glycoproteins is the CCD inhibitor cocktail offered by Mediwiss Analytic GmbH (Moers, Germany). It consists of a cocktail of three glycoproteins (bromelain, horseradish peroxidase, and ascorbate oxidase) and is mixed with twice the volume of serum to give a total inhibitor protein concentration of about $300 \mu \mathrm{g} / \mathrm{mL}$. Concerns about the rather high amount of plant protein and about the use of an active protease may be expressed.

An example for a protease treated inhibitor is the "proglycan" CCD-blocker (www.proglycan.com) consisting of bromelain glycopeptide coupled to human serum albumin [20]. This semi-synthetic inhibitor is added to serum in a volume ratio of $1: 50$ to arrive at a final concentration of $20 \mu \mathrm{g} / \mathrm{mL}$. Extensive tests were performed with allergen strips (Fig. 3), the ImmunoCAP single allergen system, the ImmunoCAP ISAC (both Phadia, Uppsala, Sweden), and the Immulite 2000 (Siemens Healthcare, Erlangen, Germany) [20]. Generally, CCD inhibition led to a vast reduction of the number of positive, recte false-positive results in all test systems, even in the ISAC system for the reasons detailed above. Unsatisfactory results where the reading remained above or only slightly below the threshold of $0.35 \mathrm{U} / \mathrm{mL}$ were notably obtained for honeybee venom and the venom component Api m 1 (Tab. 1).
The positive reaction of Api $\mathrm{m} 1$ in the single allergen test remained an enigma as the same component did not show any IgE binding in ISAC, which is much more in line with recombinant Api $\mathrm{m} 1$ not being glycosylated. Could the cotton glycoprotein problem be responsible for such a high reading? More interesting is the case of the two in sect venom extracts (aka complete allergens), where the non-inhibited result did not allow to decide on the culprit insect. The CCD-inhibited readings still saw honeybee venom as positive, but the difference to wasp venom now was huge (Tab. 1). The remaining $1.1 \mathrm{U} / \mathrm{mL}$ could arise from incomplete inhibition owed to the fact that bee venom has MMF and MMFF structures rather than MUXF (Fig. 1) as found on the semi-synthetic CCD-blocker [20]. Given the homology between bee and wasp venom proteins, the $1.1 \mathrm{U} / \mathrm{mL}$ might also result from cross-reactivity of a part of the anti-wasp venom IgE population. If this diagnosis was performed to decide on which venom to use for specific immune therapy, the result could hardly be clearer.

This review is not the place to discuss economic aspects or the difficulties of interpreting array data. It shall nevertheless be pointed out that combining extract-based sIgE determinations with CCD-inhibitions has the potential to resolve ambiguities arising from polysensitization.
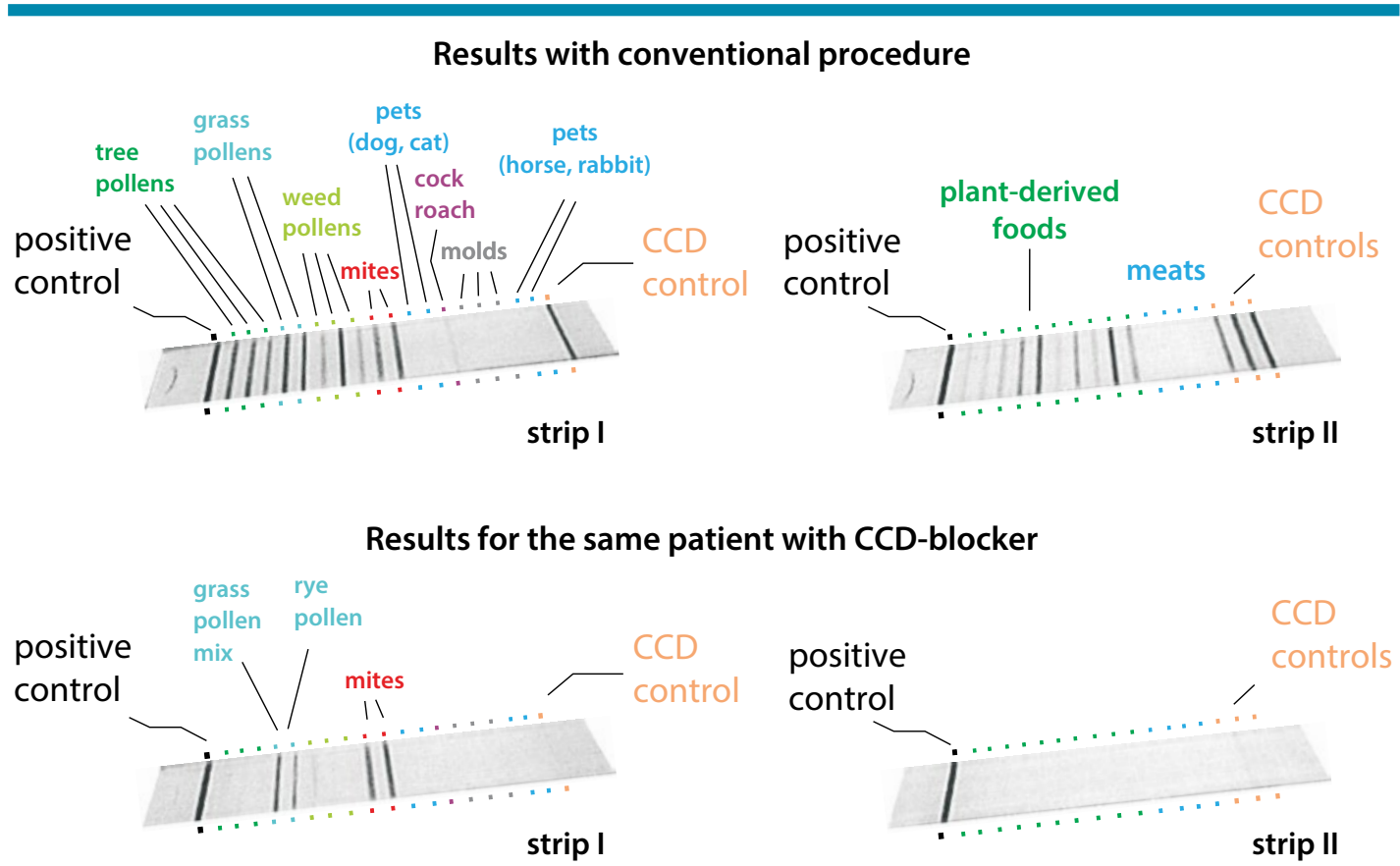

Fig. 3: Example of the effect of CCD inhibition. Serum of a 46-year-old male from Carinthia, Austria, was tested with custom made multi-allergen strips (Mediwiss Analytics, Moers, Germany) containing one or three CCD reporter bands. Experimental details can be found in [20]. In the presence of inhibitor only allergens with anamnestic substantiation appeared as positive. 


\section{Alpha-1,3-galactose - a mammalian CCD}

A decade ago, two at first independent observations led to the discovery of yet another crossreactive carbohydrate structure: allergic reactions towards a glycoprotein drug and delayed anaphylaxis after meat consumption. Severe, even lethal anaphylactic reactions were caused by the then novel anti-cancer antibody Cetuximab [30]. It was concluded that these reactions were based on pre-existing IgE against the alpha-galactose epitope (Gal- $\alpha-1,3-$ Gal- $\beta-1,4-G l c N A c)$ [30]. While antibodies against this epitope prevail in the population [31], heavy adverse effect against Cetuximab were only seen in certain areas of USA's Midwest (later also Kenya) and the current guess for this phenomenon is sensitization by tick bites [32].

At the same place and time episodes of meat allergy were observed, which appeared strange as the clinical symptoms developed only several hours after the carnal meal and as they sprang up without warning and re-appeared unpredictably [33]. With the raised awareness due to the Cetuximab case, IgE to alpha-Gal was identified as the likely culprit epitope of meat allergy [32]. Pork kidney turned out as a particularly potent elicitor of anaphylactic reactions [34]. There appears to be a correlation between meat allergy and alpha-Gal IgE that allows to regard alpha-Gal as a carbohydrate epitope with clinical relevance $[34,35]$. It should nevertheless be added that alpha-Gal IgE can also be found in healthy individuals [Soukop K, Altmann F, Hemmer W, Vienna, Austria; unpublished results]. While most people exhibit IgG against the alpha-Gal epitope, it may require some special trigger to obtain sensitization and IgE production [36]. This trigger could come from ticks, an idea that is further substantiated by patients' history and tick habitat range in eastern Austria [Swoboda I, Vienna, Austria; personal communication].

It must be stated that the macro-structure of an alpha-Gal IgE epitope (e.g., the effect of the number of alpha-Gal residues per glycan or its occurrence on $\mathrm{O}$-glycans and glycolipids in addition to $\mathrm{N}$-glycans) has not yet been defined [32]. Skin tests with an alpha-Gal containing non-allergenic protein have not yet been performed. The use of Cetuximab and bovine thyroglobin appears as a rather awkward approximation to a specific and defined tool for anti alpha-Gal-IgE determination or inhibition as both are a mixture of various structures and both contain $\mathrm{N}$-glycolylneuraminic acid, another potential IgE epitope. Neo-glycoproteins with various trisaccharides containing $\alpha-1,3$ galactose (Dextra Laboratories, Reading, United Kingdom) may be useful but have not been tested,
Tab. 1: Serum of a 46-year-old male analyzed by various methods with and without application of a CCD inhibitor (Extracted from [20])**

\begin{tabular}{|l|l|l|l|}
\hline CAP with extracts & $\begin{array}{l}\text { CAP } \mathbf{n} \\
\text { U/mL }\end{array}$ & $\begin{array}{l}\text { CAP i } \\
\text { U/mL }\end{array}$ & Evaluation \\
\hline Bee venom & 28.5 & 1.1 & drastic reduction, still pos. \\
\hline Yellow jacket venom & 28.5 & 18.3 & clearly positive \\
\hline
\end{tabular}

CAP with components

\begin{tabular}{|l|l|l|l|}
\hline rApi m1 & 1.63 & 0.31 & conflict with ISAC \\
\hline rVes v1 & 11.8 & 9.7 & clearly positive \\
\hline rVes v5 & 48.7 & 44.6 & clearly positive \\
\hline ISAC (selected results) & ISAC n & ISAC $\mathbf{~}$ & \\
\hline rApi m1 & 0 & 0 & clearly negative \\
\hline rPol d5 & 1.0 & 1.5 & clearly positive \\
\hline rVes v5 & 6.2 & 8.9 & clearly positive
\end{tabular}

*Sera were tested by the either the conventional ImmunoCAP test (Phadia, Uppsala, Sweden) or the multi-component array ImmunCAPISAC (Phadia, Uppsala, Sweden) either in the normal format $(C A P n, I S A C n)$ or with CCD-inhibitor (CAP i, ISAC i).

certainly not in comparison to $\mathrm{N}$-glycans with one or two a-1,3-galactose residues (Fig. 1).

\section{Other potential CCDs}

A variety of other non-human carbohydrate determinants could emerge as cross-reactive carbohydrate determinants. But so far, neither N-glycolylneuraminic acid (as found on Cetuximab [37], nor the Lewis a epitope found on plant glycoproteins, nor arabinogalactans on allergens [38] have as yet been described as IgE epitopes, even less as crossreactive carbohydrate determinants. $B$-Arabinoses on weed pollen allergens can bind IgE but do not seem to be widely occurring [38].

\section{Conclusion}

Specificity of diagnostic tests is a big issue. Thus, it is surprising that in the case of CCDs the maximum achievement so far are products for the detection of anti-CCD IgE. Obviously, there is little pressure to react. Indeed, in the case of single allergen testing both physician and patient are contented if the four or five allergens tested provide positive results. The physician was - apparently - right and the patient is impressed by her/ his uncanny instinct. Should the "allergen" later on never cause any discomfort it is taken by the 
patient as a confirmation of the treatment's quality. With multi-allergen tests, however, the need for a rational management of the CCD issue arose. Hopefully, this review facilitates dealing with CCD-based false polysensitization.

\section{Dr. Friedrich Altmann}

Department of Chemistry

University of Natural Resources and Life Sciences, Vienna

Muthgasse 18

1190 Vienna

Austria

E-Mail: friedrich.altmann@boku.ac.at

\section{Acknowledgment}

The guiding advises of Ines Swoboda (Campus Vienna Biocenter, Vienna, Austria) and Wolfgang Hemmer (Floridsdorf Allergy Center, Vienna, Austria) are gratefully acknowledged.

\section{Conflict of interest}

The author declares that there are no conflicts of interest.

\section{Cite this as}

Altmann F. Coping with cross-reactive carbohydrate determinants in allergy diagnosis. Allergo J Int 2016;25: 98-105

\section{DOI: $10.1007 / \mathrm{s} 40629-016-0112-6$}

\section{References}

1. Ebo DG, Hagendorens MM, Bridts $\mathrm{CH}$, De Clerck LS, Stevens WJ. Sensitization to cross-reactive carbohydrate determinants and the ubiquitous protein profilin: mimickers of allergy. Clin Exp Allergy 2004:34:137-44

2. Aalberse RC, Koshte V, Clemens JG. Immunoglobulin E antibodies that crossreact with vegetable foods, pollen, and Hymenoptera venom. J Allergy Clin Immunol 1981;68:356-64

3. Kurosaka A, Yano A, Itoh N, Kuroda Y, Nakagawa T, Kawasaki T. The structure of a neural specific carbohydrate epitope of horseradish peroxidase recognized by anti-horseradish peroxidase antiserum. J Biol Chem 1991;266:4168-72

4. Ishihara H, Takahashi N, Oguri S, Tejima S. Complete structure of the carbohydrate moiety of stem bromelain. An application of the almond glycopeptidase for structural studies of glycopeptides. J Biol Chem 1979;254:10715-9

5. Kubelka V, Altmann F, Staudacher E, Tretter V, Marz L, Hard K, et al. Primary structures of the N-linked carbohydrate chains from honeybee venom phospholipase A2. Eur J Biochem 1993;213:1193-204

6. Tretter V, Altmann F, Kubelka V, Marz L, Becker WM. Fucose alpha 1,3-linked to the core region of glycoprotein $\mathrm{N}$-glycans creates an important epitope for IgE from honeybee venom allergic individuals. Int Arch Allergy Immunol 1993:102:259-66

7. Bencurova M, Hemmer W, Focke-TejkI M, Wilson IB, Altmann F. Specificity of IgG and IgE antibodies against plant and insect glycoprotein glycans determined with artificial glycoforms of human transferrin. Glycobiology 2004;14:457-66

8. Jin C, Hantusch B, Hemmer W, Stadlmann J, Altmann F. Affinity of IgE and IgG against cross-reactive carbohydrate determinants on plant and insect glycoproteins. J Allergy Clin Immunol 2008:121:185-90 e2
9. Jin C, Bencurova M, Borth N, Ferko B, Jensen-Jarolim E, Altmann $\mathrm{F}$, et al. Immunoglobulin $\mathrm{G}$ specifically binding plant $\mathrm{N}$-glycans with high affinity could be generated in rabbits but not in mice. Glycobiology 2006;16:349-57

10. Foetisch K, Westphal S, Lauer I, Retzek M, Altmann F, Kolarich $D$, et al. Biological activity of IgE specific for cross-reactive carbohydrate determinants. J Allergy Clin Immunol 2003;111:889-96

11. Wicklein D, Lindner B, Moll H, Kolarich D, Altmann F, Becker $W M$, et al. Carbohydrate moieties can induce mediator release: a detailed characterization of two major timothy grass pollen allergens. Biol Chem 2004;385:397-407

12. Mertens M, Amler S, Moerschbacher BM, Brehler R. Cross-reactive carbohydrate determinants strongly affect the results of the basophil activation test in hymenoptera-venom allergy. Clin Exp Allergy 2010;40:1333-45

13. van der Veen MJ, van Ree R, Aalberse RC, Akkerdaas J, Koppelman SJ, Jansen HM, et al. Poor biologic activity of cross-reactive IgE directed to carbohydrate determinants of glycoproteins. J Allergy Clin Immunol 1997;100:327-34

14. Wilson IB, Zeleny R, Kolarich D, Staudacher E, Stroop CJ, Kamerling JP, et al. Analysis of Asn-linked glycans from vegetable foodstuffs: widespread occurrence of Lewis a, core alpha1,3-linked fucose and xylose substitutions. Glycobiology 2001;11:261-74

15. Paulus KE, Mahler V, Pabst M, Kogel KH, Altmann F, Sonnewald U. Silencing beta1,2-xylosyltransferase in Transgenic Tomato Fruits Reveals xylose as Constitutive Component of Ige-Binding Epitopes. Front Plant Sci 2011;2:42

16. Mari A. IgE to cross-reactive carbohydrate determinants: analysis of the distribution and appraisal of the in vivo and in vitro reactivity. Int Arch Allergy Immunol 2002;129:286-95

17. Vidal C, Sanmartin C, Armisen M, Rodriguez V, Linneberg A, Gonzalez-Quintela A. Minor interference of cross-reactive carbohydrates with the diagnosis of respiratory allergy in standard clinical conditions. Int Arch Allergy Immunol 2012;157:176-85

18. Vidal C, Vizcaino L, Diaz-Peromingo JA, Garrido M, Gomez-Rial J, Linneberg A, et al. Immunoglobulin-E reactivity to a glycosylated food allergen (peanuts) due to interference with cross-reactive carbohydrate determinants in heavy drinkers. Alcohol Clin Exp Res 2009:33:1322-8

19. Amoah AS, Obeng BB, Larbi IA, Versteeg SA, Aryeetey $Y$, Akkerdaas $\mathrm{JH}$, et al. Peanut-specific IgE antibodies in asymptomatic Ghanaian children possibly caused by carbohydrate determinant cross-reactivity. J Allergy Clin Immunol 2013;132:639-47

20. Holzweber F, Svehla E, Fellner W, Dalik T, Stubler S, Hemmer W, et al. Inhibition of IgE binding to cross-reactive carbohydrate determinants enhances diagnostic selectivity. Allergy 2013;68:1269-77

21. Aalberse RC. Clinical relevance of carbohydrate allergen epitopes. Allergy 1998;53:54-7

22. Hemmer W, Focke M, Kolarich D, Wilson IB, Altmann F, Wohrl S, et al. Antibody binding to venom carbohydrates is a frequent cause for double positivity to honeybee and yellow jacket venom in patients with stinging-insect allergy. J Allergy Clin Immunol 2001;108:1045-52

23. Jin C, Focke M, Leonard R, Jarisch R, Altmann F, Hemmer $W$. Reassessing the role of hyaluronidase in yellow jacket venom allergy. J Allergy Clin Immunol 2010;125:184-90 e1

24. Sturm GJ, Jin C, Kranzelbinder B, Hemmer W, Sturm EM Griesbacher A, et al. Inconsistent results of diagnostic tools hamper the differentiation between bee and vespid venom allergy. Plos One 2011;6:e20842

25. Treudler R, Simon JC. Overview of component resolved diagnostics. Curr Allergy Asthma Rep 2013;13:110-7

26. Ebo DG, Van Vaerenbergh $M$, de Graaf DC, Bridts $C H$, De Clerck LS, Sabato V. In vitro diagnosis of Hymenoptera 
venom allergy and further development of component resolved diagnostics. Expert Rev Clin Immunol

2014; 10:375-84

27. Hamilton RG, Kleine-Tebbe J. Molecular Allergy Diagnostics: Analytical Features That Support Clinical Decisions. Curr Allergy Asthma Rep 2015;15:556

28. Huss-Marp J, Gutermuth J, Schäffner I, Darsow U, Pfab F, Brockow K, et al. Vergleich molekularer und extraktbasierter lgE-Diagnostik mittels Multiplex- und Singleplex-Test. Allergo J Int 2015;24:46-53

29. Hemmer W, Holzweber F, Wöhrl S, Wantke F, Altmann F. ImmunoCAP Cellulose Displays Cross-Reactive Carbohydrate Epitopes and May Cause False-Positive Test Results in Patients with anti-CCD IgE Antibodies. J Allergy Clin Immunol 2014;133:1

30. Chung CH, Mirakhur B, Chan E, Le QT, Berlin J, Morse M, et al. Cetuximab-induced anaphylaxis and IgE specific for galactose-alpha-1,3-galactose. N Engl J Med 2008;358:1109-17

31. Macher BA, Galili U. The Galalpha1,3Galbeta1,4GIcNAc-R (alpha-Gal) epitope: a carbohydrate of unique evolution and clinical relevance. Biochim Biophys Acta 2008; 1780:75-88

32. Steinke JW, Platts-Mills TA, Commins SP. The alpha-gal story: lessons learned from connecting the dots. J Allergy Clin Immunol 2015;135:589-96; quiz 97
33. Commins SP, Satinover SM, Hosen J, Mozena J, Borish L, Lewis BD, et al. Delayed anaphylaxis, angioedema, or urticaria after consumption of red meat in patients with IgE antibodies specific for galactose-alpha-1,3-galactose. J Allergy Clin Immunol 2009;123:426-33

34. Fischer J, Hebsaker J, Caponetto P, Platts-Mills TA, Biedermann T. Galactose-alpha-1,3-galactose sensitization is a prerequisite for pork-kidney allergy and cofactor-related mammalian meat anaphylaxis. J Allergy Clin Immunol 2014;134:755-9 e1

35. Rispens T. IgE antibodies specific to alpha-gal: an example of clinically relevant cross-reactive anti-carbohydrate antibodies. Clinical and Experimental Allergy 2014;44:1008-11

36. Rispens T, Derksen NIL, Commins SP, Platts-Mills TA, Aalberse RC. IgE Production to alpha-Gal Is Accompanied by Elevated Levels of Specific IgG1 Antibodies and Low Amounts of IgE to Blood Group B. Plos One 2013;8(2):e55566

37. Stadlmann J, Pabst M, Kolarich D, Kunert R, Altmann F. Analysis of immunoglobulin glycosylation by LC-ESI-MS of glycopeptides and oligosaccharides. Proteomics 2008;8:2858-71

38. Leonard R, Wopfner N, Pabst M, Stadlmann J, Petersen $\mathrm{BO}$, Duus JO, et al. A new allergen from ragweed (Ambrosia artemisiifolia) with homology to art $\mathrm{v} 1$ from mugwort. J Biol Chem 2010;285:27192-200 